Agronomía Costarricense 39(2): 33-45. ISSN:0377-9424 / 2015

www.mag.go.cr/rev_agr/index.html www.cia.ucr.ac.cr

\title{
DIVERSIDAD GENÉTICA ENTRE SUBPOBLACIONES RACIALES BOVINAS DE COSTA RICA
}

\author{
Marco Martínez, ${ }^{*}$ Bernardo Vargas ${ }^{1 *}$, Juan Miguel Cordero $^{* *}$, Idania Chacón $^{* *}$, Bernal León $^{* *}$ \\ Palabras clave: Estructura genética; relaciones filogenéticas; microsatélites. \\ Keywords: Genetic structure; phylogenetic relationship; microsatellites.
}

Recibido: 08/04/15

Aceptado: 17/06/15

\section{RESUMEN}

El objetivo del estudio fue cuantificar la diversidad genética entre 16 subpoblaciones raciales bovinas de Costa Rica, con base en 1412 muestras de ADN bovino de todo el país, evaluadas mediante 18 marcadores microsatélites. El número promedio de alelos $(\mathrm{Na})$ por locus dentro de raza fue de 10,3 , que varían entre 8 (Holstein $\times$ Jersey) y 13 (Criolla para doble propósito). El número promedio de alelos efectivo (Ne) fue de 5,04, con cambios entre 4,18 (Jersey) y 5,64 (Bos taurus $\times$ Bos indicus). La heterocigosidad observada promedio fue de 0,77 , variando entre 0,73 (Jersey) y 0,81 (Bos taurus $\times$ Bos indicus). La heterocigosidad esperada (He) promedio fue de 0,78 , que oscilan entre 0,74 (Jersey y Holstein $\times$ Jersey) y 0,81 (Bos taurus $\times$ Bos indicus, Criolla para doble propósito y Cruces para doble propósito). El contenido de información polimórfica (PIC) fue de 0,76 , con variaciones entre 0,71 (Jersey y Holstein $\times$ Jersey) y 0,79 (Criollas para doble propósito y Cruces para doble propósito). El $F_{I S}$ promedio fue de 0,02 , con oscilaciones entre $-0,03$ (Holstein $\times$ Jersey) a 0,04 (Brahman, Criolla para carne y Cruces para leche). La desviación del equilibrio Hardy Weinberg no fue significativa $(p>0,05)$ en la mayoría de los loci para las subpoblaciones raciales. El subgrupo con mayor número de loci en desequilibrio

1 Autor para correspondencia. Correo electrónico: bernardo.vargas.leitón@una.cr

* Posgrado Regional en Ciencias Veterinarias Tropicales, Universidad Nacional, Costa Rica.

\section{ABSTRACT}

Genetic diversity among bovine racial subpopulations of Costa Rica. The objetive of this study was to quantify the genetic diversity among 16 bovine racial subpopulations of Costa Rica, based on 1412 samples of bovine DNA from around the country, which were evaluated using 18 microsatellite markers. Average number of alleles (Na) per locus within breed was 10.3, ranging from 8 (Holstein $\times$ Jersey) to 13 (Dual Purpose Creole). Average number of effective alleles (Ne) was 5.04, ranging between 4.18 (Jersey) and 5.64, (Bos taurus $\times$ Bos indicus). Average observed heterocigozity $(\mathrm{Ho})$ was 0.77 , varying between 0.73 (Jersey) and 0.81 (Bos taurus $\times$ Bos indicus). Average expected heterocigozity $(\mathrm{He})$ was 0.78 , oscillating between 0.74 (Jersey and Holstein $\times$ Jersey) and 0.81 (Bos taurus $\times$ Bos indicus, dual purpose Creole and dual purpose Crosses). The polymorphic information content (PIC) was 0.76 , ranging between 0.71 (Jersey and Holstein $\times$ Jersey) and 0.79 (dual purpose Creole and dual purpose crosses). Average $F_{I S}$ was 0.02 , ranging from -0.03 (Holstein $\times$ Jersey) to 0.04 (Brahman, Beef Creole and Dairy crosses). Deviation from Hardy Weinberg equilibrium was not significant $(p>0.05)$ for the majority of loci within racial subpopulations. Subgroup with the highest number of loci in disequilibrium was ** Servicio Nacional de Salud Animal, Heredia,
Costa Rica. 
fue Jersey (8 loci), mientras que los subgrupos Bos taurus $\times$ Bos indicus, Criolla para leche $\mathrm{y}$ Holstein $\times$ Jersey presentaron solo 1 locus en desequilibrio. Los índices de fijación $F_{I S}(0,02)$, $F_{I T}(0,05)$ y $F_{S T}(0,03)$ indicaron cierta tendencia hacia la homocigosidad. Los dendrogramas mostraron 3 agrupaciones raciales claramente diferenciadas que coinciden con las razas de origen Bos taurus, Bos indicus y sus respectivos cruces. Los resultados del análisis indicaron que el número de microsatélites empleados sí permitió establecer una discriminación clara a nivel de las frecuencias alélicas y en la distribución del tamaño de los alelos entre las subpoblaciones de distintas especies y aún entre razas puras.

\section{INTRODUCCIÓN}

La ganadería bovina en Costa Rica inicia su desarrollo a partir de 1561, cuando ingresaron los primeros bovinos de origen europeo. En 1568, el gobernador de Costa Rica Perafán de Rivera ordenó la importación de 400 bovinos de Honduras y de ahí se fundaron los primeros hatos de cría en el país (Quirós 2006). En los siglos posteriores se dio la evolución de diferentes sistemas de producción en distintas regiones del país y el ingreso de diversos tipos raciales adecuados para diferentes fines: leche carne o doble propósito. Según el último censo ganadero se registró en Costa Rica una población bovina de 1358209 cabezas de las cuales un 70,3\%, (955 854 cabezas) eran hembras y un 29,6\% (402 355 cabezas) eran machos (CORFOGA 2000).

Los estudios de genética poblacional ayudan a cuantificar la diversidad genética presente en una población. Las relaciones genéticas entre diferentes subpoblaciones se pueden inferir a partir de los datos de las frecuencias de los alelos. Recientemente, los datos del ADN de microsatélites son ampliamente utilizados para los estudios
Jersey (8 loci), while subgroups Bos taurus $\times$ Bos indicus, Dairy Creole and Holstein $\times$ Jersey showed only 1 locus in disequilibrium. Fixation indexes $F_{I S}(0.02), F_{I T}(0.05)$ and $F_{S T}(0.03)$ indicated some tendency towards homocigozity. The dendrograms showed 3 distinct racial groups that match races of Bos taurus, origin Bos indicus and their crosses. The results of the analysis indicated that the number of microsatellites used allowed to establish a clear discrimination at the level of the alelic frequencies and in the distribution of the size of aleles between the subpopulations of different species and even between pure races. de genética de poblaciones en diversos organismos, debido a su alto grado de polimorfismo genético (Estoup et ál. 2002).

Para comparar la diferenciación existente entre subpoblaciones raciales se pueden utilizar índices de distancia genética tales como los estadísticos $F$ (Wright 1965, Nei 1977, Weir y Cockerham 1984), que permiten establecer comparaciones, determinar relaciones filogenéticas y analizar la estructura de la población. Los estadísticos $F_{I S}$ y $F_{I T}$ explican las correlaciones entre 2 unidades gaméticas tomadas al azar de una subpoblación y del total de la población como un todo respectivamente; y nos indica el exceso o déficit de heterocigotos presentes; mientras que el $F_{S T}$ es la correlación entre 2 gametos tomados al azar de cada una de las subpoblaciones y mide el grado de diferenciación genética entre 2 subpoblaciones (Wright 1965).

Más recientemente Slatkin (1995) desarrolló la medida $R_{S T}$ que se define como la proporción de variación en la longitud de alelos de loci de microsatélites debido a las diferencias entre poblaciones. El $R_{S T}$ asume que cada mutación 
cambia una longitud de un alelo por una unidad repetida (Slatkin 1995).

En Costa Rica no se ha realizado aún ningún análisis de diversidad genética dentro de la población bovina existente. El objetivo del presente estudio es analizar la diversidad genética en subpoblaciones raciales de la población bovina de Costa Rica, por medio de marcadores microsatélites.

\section{MATERIALES Y MÉTODOS}

\section{Procedencia y estructura de la muestra}

Para la realización del presente estudio se contó con 1412 muestras de ADN bovino obtenidas en 744 hatos localizados en distintas zonas ganaderas del país (Cordero et ál. 2013). Las muestras disponibles correspondieron a ADN extraído a partir de folículos pilosos de la cola del bovino. Con base en las recomendaciones de FAO/ISAG (FAO 2011) se procuró maximizar el número de hatos visitados para evitar muestrear animales emparentados, por lo que se limitó a 2 el número de muestras colectadas por establecimiento, salvo aquellos en los cuales se encontraron múltiples tipos raciales.

Las muestras se colocaron en sobres individuales con el número de registro oficial del establecimiento y el sistema de producción según el Sistema Integrado de Registro de Establecimientos Agropecuarios (SIREA 2013), y la identificación del animal dentro de la finca, tipo racial del animal, sexo y edad. El tipo racial se estableció de manera aproximada con base en apreciaciones visuales. Debido a la alta variabilidad racial encontrada, se realizó una clasificación por tipos raciales afines (Cuadro 1), de lo cual se obtuvo finalmente un total de 16 grupos. El razonamiento utilizado para realizar esta agrupación detalla a continuación.

Cuadro 1. Número de animales muestreados por raza.

\begin{tabular}{|c|c|c|c|c|}
\hline Subpoblación & Tipo Racial & Nomenclatura & Número de animales & $\%$ \\
\hline 1 & Bos indicus & B_ind & 97 & 6,9 \\
\hline 2 & Brahman & Brahman & 263 & 18,6 \\
\hline 3 & Bos taurus & B_tau & 22 & 1,6 \\
\hline 4 & Bos taurus $\times$ Bos indicus & Btau $\times$ Bind & 43 & 3,0 \\
\hline 5 & Criollas para Carne & Criolla_Car & 103 & 7,3 \\
\hline 6 & Criollas para Doble Propósito & Criolla_Dpr & 189 & 13,4 \\
\hline 7 & Criollas para leche & Criolla_Lec & 39 & 2,8 \\
\hline 8 & Cruces para Carne & Cruce_Car & 68 & 4,8 \\
\hline 9 & Cruces para Doble Propósito & Cruce_Dpr & 95 & 6,7 \\
\hline 10 & Cruces para leche & Cruce_Lec & 64 & 4,5 \\
\hline 11 & Guernsey & Guernsey & 49 & 3,5 \\
\hline 12 & Holstein & Holstein & 89 & 6,3 \\
\hline 13 & Holstein $\times$ Jersey & Hol $\times$ Jer & 34 & 2,4 \\
\hline 14 & Jersey & Jersey & 158 & 11,2 \\
\hline 15 & Pardo Suizo & Pardo Suizo & 49 & 3,5 \\
\hline \multirow[t]{2}{*}{16} & Simmental & Simmental & 50 & 3,5 \\
\hline & Población Total & & 1412 & 100 \\
\hline
\end{tabular}


El grupo clasificado como Bos Indicus (subpoblación 1, Cuadro 1) estuvo conformado mayormente por animales reportados como de tipo "cebú" y algunas razas presentes en números muy reducidos, tales como la Indubrasil, Gyr o Nellore. La subpoblación 2 estuvo conformada por animales reportados como "Brahman", aunque esta denominación en muchos casos corresponde a animales que no son de raza pura. $\mathrm{La}$ subpoblación 3 agrupó principalmente animales reportados como cruces entre razas Holstein, Pardo Suizo, Guernsey o Jersey, y algunos pocos animales de raza Chiannina y Hereford. El grupo Bos taurus $\times$ Bos indicus (subpoblación 4 , Cuadro 1) incluyó principalmente cruces entre razas taurinas como la Holstein, Pardo Suizo o Jersey, con otras cebuinas como la Brahman.

En muchos casos el grupo racial se reportó originalmente como "Criollo". Cabe aclarar que esta denominación es utilizada actualmente en Costa Rica de manera un tanto arbitraria, ya que no corresponde a la definición real del término Criollo, la cual hace alusión a grupos raciales descendientes del ganado tipo Bos taurus procedente de la península Ibérica en el siglo XVI (FAO 2012, Quirós 2006). En la práctica, el término es actualmente utilizado de manera indistinta para describir cruces indefinidos entre razas Bos taurus $\times$ Bos indicus, o inclusive para describir ganado tipo Bos indicus de raza indefinida. Por esta razón, para el presente estudio el tipo racial reportado como Criollo se subdividió en 3 grupos en función del sistema de producción (subpoblaciones 5, 6, y 7; Cuadro 1). Lo mismo sucedió con animales reportados originalmente como "Cruce", ya que estos animales son producto de una mezcla indefinida de razas. Para el presente estudio estos casos también fueron clasificados en 3 grupos (subpoblaciones 8, 9 y 10; Cuadro 1) en función del sistema de producción (Carne, Leche o Doble Propósito).

La extracción de ADN del folículo piloso se realizó en el área de Biología Molecular y Genética del Laboratorio de Bioseguridad del SENASA a partir de una muestra de mechones de pelo de la cola con folículo, según el procedimiento descrito por Cordero et ál. (2013). Se analizó un conjunto de 18 marcadores, entre ellos 15 de los recomendados por FAO/ISAG (FAO 2011) para uso en bovinos: TGLA227, BM2113, TGLA53, ETH10, SPS115, TGLA126, TGLA122, INRA23, ETH3, ETH225, BM1824, BM1818, CSRM60, CSSM66 y ILSTS006, más otros 3 marcadores adicionales: SPS113, RM067 y MGTG4B. Los productos de la amplificación fueron sometidos a electroforesis capilar según el procedimiento descrito por Cordero et ál. (2013). Los polimorfismos de los microsatélites fueron discriminados de acuerdo con sus patrones de fluorescencia y tamaño. Los datos se visualizaron e identificaron mediante el software GeneMapper v3.0 (Applied Byosistems 2002).

\section{Cálculo de parámetros de diversidad genética por subgrupo racial}

Con base en la información obtenida de los análisis de ADN se estimaron los siguientes parámetros genéticos por subgrupo racial: el número total de alelos $(\mathrm{Na})$ observados; el número efectivo $(\mathrm{Ne})$ de alelos, obtenido como $N e=1 / \Sigma \mathrm{p}^{2}$ con $\mathrm{p}=$ frecuencias alélicas por marcador microsatélite; el nivel de Heterocigosidad observada $(\mathrm{Ho})$, obtenido como la proporción observada de individuos heterocigóticos; el nivel de Heterocigosidad esperada (He), obtenido como $\left(\mathrm{He}=1-\sum_{i=1}^{n} P_{1}^{2}\right)$ donde pi...pn son las frecuencias de $\operatorname{los} n$ alelos; el Contenido de Información Polimórfica $\left(C I P=1-\sum_{i=1}^{n} P_{1}^{2}-\sum_{i=1}^{n} \sum_{j=i=1}^{n} 2 P_{i}^{2} P_{j}^{2}\right)$ $\mathrm{y}$ el Coeficiente de Consanguinidad $\left(F_{I S}=\frac{(H e-H o)}{H e}\right)$. Además se compararon las frecuencias alélicas observadas contra las frecuencias alélicas esperadas bajo equilibrio Hardy-Weinberg por medio de la prueba Chicuadrado $\left(X^{2}=\sum \frac{\left(f_{o}-f_{e}\right)^{2}}{f_{e}}\right)$ donde $\chi^{2}=$ estadístico de prueba; $f_{o}=$ frecuencias observadas y $f_{e}$ $=$ frecuencias esperadas. Los cálculos anteriores se realizaron mediante el software especializado GenAlEx v.6.50 (Peakall y Smouse 2006, 2012) e Info-Gen (Balzarini y Di Rienzo 2004). 


\section{Análisis de estructura genética}

Se realizó un Análisis de Varianza Molecular (AMOVA, Excoffier et ál. 1992) mediante el programa GenAlEx v6.5 (Peakall y Smouse 2006, 2012) con el fin de evaluar la existencia de diferenciación genética significativa entre las subpoblaciones bovinas formadas por los distintos grupos raciales identificados. Mediante este análisis se obtuvieron estimados de las varianzas genéticas entre razas (Ver), entre individuos (Vei) e intra-individuos (Vii). A partir de estos se calcularon los estadísticos $F$ (Wright 1950), específicamente, $F_{S T}=\operatorname{Ver} /(\mathrm{Vii}+\mathrm{Vei}+\mathrm{Ver}), F_{I S}$ $=V e i /(V i i+V e i)$ y $F_{I T}=(V e i+V e r) /(V i i+V e i$ $+V e r$ ), para los cuales se obtuvo un estimado de significancia estadística mediante análisis de remuestreo (Felsenstein 1985) con 1000 permutaciones aleatorias.

Se calcularon además las distancias genéticas entre todos los pares de razas con 2 métodos diferentes: la distancia estándar (DS) de Nei (Nei 1972), calculada como () donde $P x$ y $P y$ son las homocigosis medias sobre loci en la población $\mathrm{X}$ e Y, respectivamente; y los estimados de diferenciación genética $R_{S T}$ (Slatkin 1995), que se obtuvieron mediante la fórmula: $\left(R_{S T}=\frac{V_{E R}}{\left(V_{I I}+V_{E I}+V_{E R}\right)}\right)$, donde $V_{E R}=$ varianza entre razas, $V_{I I}=$ varianza intra-individuos y $V_{E I}=$ varianza entre individuos. Ambos estimados de distancia genética se calcularon mediante el programa GenAlEx v.6.5 (Peakall y Smouse 2006,2012 ). Para $R_{S T}$ se evaluó la significancia estadística mediante análisis de remuestreo (Felsenstein 1985) con 1000 permutaciones aleatorias.
Para visualizar las relaciones genéticas entre las distintas subpoblaciones raciales, se construyó un dendrograma según el algoritmo Neighbor Joining (NJ) (Saitou y Nei 1987) con la medida de distancia estándar de Nei, según se implementa en el programa Poptree2 (Takezaki et ál. 2010). La consistencia de las ramificaciones del dendrograma se evaluó mediante análisis de remuestreo (Felsenstein 1985) con 1000 permutaciones. Para efectos comparativos se generó un segundo dendrograma con el algoritmo de Ward (Ward 1963) con distancias basadas en el índice de similitud de Dice (Dice 1945), por medio del programa Info-Gen (Balzarini y Di Rienzo 2004).

\section{RESULTADOS Y DISCUSIÓN}

\section{Diversidad genética dentro de los subgrupos raciales}

El número promedio de alelos $(\mathrm{Na})$ por locus en las subpoblaciones raciales fue de 10,3 (Cuadro 2). Los valores obtenidos variaron desde un mínimo de 8 alelos (Holstein $\times$ Jersey) hasta un máximo de 13 alelos (Criollas para doble propósito). En lo que se refiere al valor de $\mathrm{Ne}$, el promedio para todas las subpoblaciones raciales fue de 5,04. Los valores más bajos de $\mathrm{Ne}$ los presentaron las subpoblaciones Jersey $(4,18)$ y Holstein $\times$ Jersey $(4,22)$; mientras que las subpoblaciones Criollas para doble propósito y Bos taurus $\times$ Bos indicus presentaron los valores más altos de $\mathrm{Ne}$ (5,63 y 5,64 respectivamente). 
Cuadro 2. Número total $(\mathrm{Na})$ y efectivo $(\mathrm{Ne})$ de alelos, heterocigosis observada ( $\mathrm{Ho}$ ) y esperada $(\mathrm{He})$, contenido de información polimórfica $(C I P)$, coeficiente de endogamia $\left(F_{I S}\right)$ y número de marcadores según valor de significancia ${ }^{1}$ de la prueba Hardy-Weinberg $\left(\operatorname{Prob}>\mathrm{X}^{2}\right)$ para cada uno de los subgrupos raciales.

\begin{tabular}{|c|c|c|c|c|c|c|c|c|c|c|}
\hline \multirow[t]{2}{*}{ Razas $^{2}$} & \multirow[t]{2}{*}{$\mathrm{Na}$} & \multirow[t]{2}{*}{$\mathrm{Ne}$} & \multirow[t]{2}{*}{ Ho } & \multirow[t]{2}{*}{$\mathrm{He}$} & \multirow[t]{2}{*}{ CIP } & \multirow{2}{*}{$\mathrm{F}_{\mathrm{IS}}$} & \multicolumn{4}{|c|}{ Número de Marcadores según Valor P } \\
\hline & & & & & & & $(\mathrm{p}<0,05)$ & $(\mathrm{p}<0,01)$ & $(\mathrm{p}<0,001)$ & $(\mathrm{p}>0,05)$ \\
\hline B_ind & 11 & 4,67 & 0,74 & 0,76 & 0,73 & 0,02 & & 1 & 1 & 16 \\
\hline Brahman & 12 & 4,99 & 0,75 & 0,78 & 0,75 & 0,04 & 2 & 2 & 1 & 13 \\
\hline B_tau & 9 & 5,11 & 0,77 & 0,79 & 0,76 & 0,02 & 2 & & & 16 \\
\hline Btau $\times$ Bind & 10 & 5,64 & 0,81 & 0,81 & 0,78 & 0,00 & & 1 & & 17 \\
\hline Criolla_Car & 11 & 5,40 & 0,76 & 0,80 & 0,78 & 0,04 & 1 & & 2 & 15 \\
\hline Criolla_Dpr & 13 & 5,63 & 0,79 & 0,81 & 0,79 & 0,02 & 1 & 1 & 4 & 12 \\
\hline Criolla_Lec & 9 & 5,04 & 0,78 & 0,78 & 0,76 & 0,00 & & 1 & & 17 \\
\hline Cruce_Car & 11 & 5,17 & 0,77 & 0,79 & 0,77 & 0,03 & & & 3 & 15 \\
\hline Cruce_Dpr & 11 & 5,56 & 0,79 & 0,81 & 0,79 & 0,03 & & 2 & 4 & 12 \\
\hline Cruce_Lec & 10 & 5,53 & 0,77 & 0,80 & 0,78 & 0,04 & & 1 & 2 & 15 \\
\hline Guernsey & 9 & 4,43 & 0,76 & 0,76 & 0,73 & 0,00 & 1 & 1 & & 16 \\
\hline Holstein & 10 & 4,88 & 0,77 & 0,78 & 0,75 & 0,01 & 2 & & 2 & 14 \\
\hline Hol $\times$ Jer & 8 & 4,22 & 0,77 & 0,74 & 0,71 & $-0,03$ & 1 & & & 17 \\
\hline Jersey & 10 & 4,18 & 0,73 & 0,74 & 0,71 & 0,01 & 1 & 2 & 5 & 10 \\
\hline Pardo Suizo & 10 & 5,26 & 0,77 & 0,80 & 0,77 & 0,03 & & 2 & 1 & 15 \\
\hline Simmental & 10 & 4,89 & 0,77 & 0,78 & 0,75 & 0,01 & 2 & & & 16 \\
\hline Promedio & 10,3 & 5,04 & 0,77 & 0,78 & 0,76 & 0,02 & & & & \\
\hline
\end{tabular}

${ }^{1}$ Significativo $(\mathrm{p}<0,05 ; \mathrm{p}<0,01 ; \mathrm{p}<0,001) ; \mathrm{NS}=$ No significativo $(\mathrm{p}>0,05)$.

${ }^{2}$ B_ind: Bos indicus, B_tau: Bos taurus, Btau $\times$ Bind: Bos taurus $\times$ Bos indicus, Criolla_Car: Criollas para Carne, Criolla_ Dpr: Criollas para Doble Propósito, Criolla_Lec: Criollas para leche, Cruce_Car: Cruces para Carne, Cruce_Dpr: Cruces para Doble Propósito, Cruce_Lec: Cruces para leche, Hol×Jer: Holstein $\times$ Jersey.

EI número promedio de alelos detectado en el presente estudio es mayor que el reportado en estudios similares (7,2: Lirón et ál. 2006, 8,5: Egito et ál. 2007, 6,7: Ginja et ál. 2009, 7,5: Acosta et ál. 2012). Las diferencias pueden estar ligadas al hecho de que en los estudios mencionados los muestreos fueron dirigidos a razas específicas, con un menor número de muestras y explotaciones. En el presente estudio el muestreo fue aleatorio con el fin de obtener representatividad de la estructura de la población, lo que redunda en una mayor diversidad racial y genética. Además se contó con mayor número de explotaciones muestreadas en comparación con la mayoría de estudios similares. Otro factor que puede contribuir a las diferencias observadas entre estudios es la utilización de cantidades y tipos distintos de microsatélites, si bien en la mayoría de los casos existe alta coincidencia con los marcadores recomendados por la FAO/ISAG. 
En cuanto a la heterocigosis observada $(\mathrm{Ho})$, el promedio entre razas fue de 0,77 , con un valor mínimo de 0,73 (Jersey) y un máximo de 0,81 (Bos taurus $\times$ Bos indicus). La Heterocigosis esperada $(\mathrm{He})$ promedio resultó ser de 0,78 , con variaciones entre 0,74 (Jersey y Holstein $\times$ Jersey) a 0,81 (Bos taurus $\times$ Bos indicus, Criollas para doble propósito y Cruces para doble propósito). En general las mayores heterocigosis se expresan en los tipos raciales denominados criollos o cruces, lo que es consistente con su heterogeneidad fenotípica.

Cabe destacar que en la mayoría de los grupos raciales se presentó déficit de heterocigotos $(\mathrm{Ho}<\mathrm{He})$. Este déficit de heterocigotos se interpreta generalmente como una desviación de los apareamientos aleatorios, en este caso con ligera tendencia a la consanguinidad. En el caso de las poblaciones de ganado bovino, existe un efecto marcado de la selección artificial y el control de los apareamientos realizado por los criadores, lo que sin duda puede jugar un papel importante.

En 3 subpoblaciones (Bos taurus $\times$ Bos indicus, Criollas para leche y Guernsey) los valores de $\mathrm{He}$ no difieren significativamente de $\mathrm{Ho}$, lo que sugiere un estado de equilibrio $H W$ donde los apareamientos ocurren al azar. En los sistemas ganaderos de Costa Rica las razas criollas lecheras se utilizan principalmente para establecer cruces con Bos Taurus, en especial Holstein y Jersey, y/o Bos indicus con la finalidad de obtener adaptabilidad y resistencia al medio (Vilaboa et ál. 2012). Sin embargo en otros casos se han desechado las razas criollas y han optado por el uso de otras razas o cruces Bos taurus $\times$ Bos indicus para la producción de leche, al relegar en importancia, la utilización de las razas criollas lecheras (Vilaboa et ál. 2012). En Costa Rica las razas que más se utilizan en cruzamientos para ganadería de carne son las cebuínas y Simmental y sus respectivos cruces con el fin de mejorar la calidad de la carne y pesos al destete (Hidalgo et ál. 2004).

El promedio de $H o$ obtenido en este estudio $(0,77)$ es superior a valores reportados en otros estudios similares (0,75: Lirón et ál. 2006, 0,68: Egito et ál. 2007, 0,67: Ginja et ál. 2009, 0,70: Acosta et ál. 2012). En cuanto a la He encontrada en este estudio $(0,78)$, también es superior a otros valores reportados previamente (0,73: Lirón et ál. 2006, 0,74: Egito et ál. 2007, 0,69: Ginja et ál. 2009, 0,73: Acosta et ál. 2012).

Con respecto al contenido de información polimórfica $(C I P)$, se puede observar que todos los microsatélites utilizados estuvieron en la categoría de altamente informativos $(>0,50)$. El coeficiente de endogamia $\left(F_{I S}\right)$ presentó un valor promedio de 0,02 , que osciló entre $-0,03$ (Holstein $\times$ Jersey) a 0,04 (Brahman, Criollas para carne y Cruces para leche). Un $F_{I S}$ de 0 , como el encontrado en las subpoblaciones raciales Bos taurus $\times$ Bos indicus, Criollas para leche y Guernsey, significa que se encuentran en equilibrio $H W$, puesto que la heterocigosidad observada $(\mathrm{Ho})$ es semejante a la heterocigosidad esperada $(\mathrm{He})$. El resultado en esta subpoblación puede ser debido al cruce entre 2 grupos raciales, lo que expresa un exceso de heterocigotos $(\mathrm{Ho}>\mathrm{He})$ y por tanto un nivel de consanguinidad muy bajo. El valor promedio de $F_{I S}$ en este estudio es superior al $-0,03$ encontrado por Lirón et ál. (2006) y al 0,004 obtenido por Acosta et ál. (2012), e inferior al 0,09 encontrado por Egito et ál. (2007) y al 0,03 reportado por Ginja et ál. (2009).

Con respecto a la prueba de equilibrio de Hardy-Weinberg $(H W)$ la desviación del equilibrio $H W$ no fue significativa $(\mathrm{p}>0,05)$ en la mayoría de los loci (Cuadro 2) dentro de las subpoblaciones raciales. El grupo con mayor número de loci en desequilibrio $H W(\mathrm{p}<0,05)$ fue Jersey (8 loci), mientras que los grupos Bos taurus $\times$ Bos indicus, Criollas para leche $\mathrm{y}$ Holstein $\times$ Jersey presentaron solo 1 locus en desequilibrio $H W$.

\section{Análisis de estructura genética}

Según los resultados del AMOVA la proporción de la variabilidad genética atribuible a la diferencia entre las subpoblaciones raciales (Ver), entre individuos (Vei) e intra individuos (Vii) fue de $3 \%, 3 \%$ y $94 \%$ respectivamente. En estudios 
similares se han reportado mayores valores para Ver. Lirón et ál. (2006) reportaron que el 8,8\% de la variación genética total correspondió a las diferencias entre las poblaciones (razas cebú y taurinas), mientras que el 91,2\% se explicó por las diferencias entre e intra individuos. Cañón et ál. (2001) indicaron que alrededor del $7 \%$ de la variación genética total correspondió a las diferencias entre los grupos raciales, mientras que el 93\% restante correspondió a las diferencias entre e intra individuos. Por otra parte, Egito et ál. (2007) reportaron un valor aún mayor (12\%) para la variación genética atribuible a las diferencias entre los grupos raciales.

El valor menor obtenido en el presente estudio puede estar ligado nuevamente a las características del muestreo y a la clasificación de animales en grupos raciales afines. Los grupos raciales combinados (p.e criollo o cruces) están conformados por animales altamente heterogéneos, lo que magnifica la varianza dentro de grupo (entre e intra individuos) en comparación con la varianza entre los grupos (entre razas). Muchos de estos grupos además están altamente relacionados entre sí, lo que se confirma posteriormente en los dendrogramas.

La contribución de los 18 marcadores para la diferenciación de los grupos raciales fue estimada por los estadísticos $F$ (datos no mostrados). Los valores promedio de $F_{I S}, F_{I T}$ y $F_{S T}$ fueron de 0,05, 0,02 y 0,03 respectivamente. Al analizar los valores de $F_{S T}$ obtenidos para los diferentes marcadores se observó que los marcadores ETH10, ETH225 y TGLA227 son los que más contribuyen a las diferencias entre razas, mientras que los marcadores BM1818, BM1824, ILSTS006, INRA23, MGTG4B, RM067, SPS113 y TGLA53 son los que mostraron menor variabilidad. El valor promedio de $F_{S T}$ observado en el presente estudio tiende a ser inferior al obtenido en estudios similares (0,07: Jordana et ál. 2003, 0,10: Egito et ál. 2007, 0,04: Sun et ál. 2008, 0,09: Acosta et ál. 2012).

Como era de esperarse, en general se observan valores de distancias genéticas bajas entre los grupos raciales Bos indicus y sus cruces, y entre los grupos Bos taurus y sus cruces (Cuadro 3). Por el contrario, se observan distancias altas entre los grupos raciales de leche (Bos taurus) con respecto a las razas de carne (Bos indicus). Se observan además distancias reducidas entre las razas criollas y cruces de los grupos raciales anteriores.

Las distancias genéticas más bajas se obtuvieron para grupos genéticamente afines, por ejemplo valores de 0,02 se obtuvieron entre las subpoblaciones Brahman vs. Bos indicus; Criollas para doble propósito vs. Criollas para carne; Cruces para carne vs. Criollas para carne; Cruces para doble propósito vs. Criollas para carne; Cruces para carne vs. Criollas para doble propósito o Cruces para doble propósito vs. Criollas para doble propósito. La reducida distancia genética entre los grupos Criollos y los Cruces, dentro de cada sistema de producción, confirma que el término Criollo se utiliza localmente para describir ganado obtenido por cruzamientos entre razas Bos taurus o Bos indicus, en proporciones variables.

El uso incorrecto o confuso de denominaciones raciales es un problema reportado a nivel global.

Recientemente, una consultoría realizada por la comisión de recursos genéticos de la FAO propuso descontinuar el uso de términos tales como raza nativa, indígena o autóctona (FAO 2012). En su lugar, se ha sugerido que, para efectos de caracterización genética, se utilicen criterios de clasificación basados en adaptabilidad racial. Se considera una raza adaptada aquella que ha permanecido en un país por al menos 40 años o 6 generaciones, lo que se considera suficiente para adecuarse a uno o más de los sistemas tradicionales de producción (FAO 2012). En contraposición se recomienda el uso del término exótico para aquellas razas introducidas recientemente o que son importadas de manera continua.

Por el contrario, las distancias genéticas más altas se observaron entre las subpoblaciones Holstein vs. Bos indicus $(0,42)$; Jersey vs. Bos indicus $(0,45)$; Holstein $\times$ Jersey vs. Bos indicus $(0,47)$ y Guernsey vs. Bos indicus $(0,48)$. Estos 
MARTÍNEZ et ál.: Subpoblaciones raciales bovinas en Costa Rica

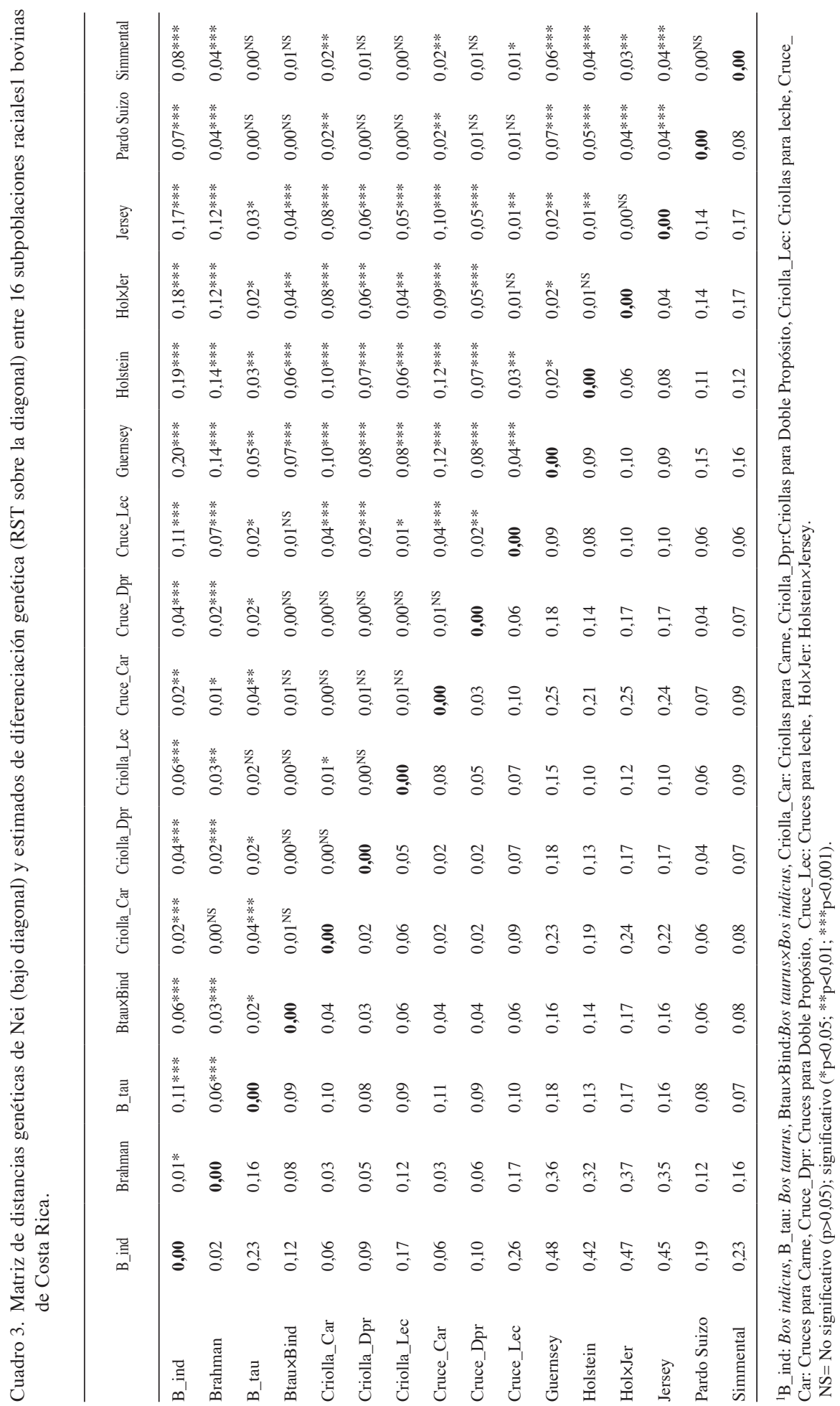

Agronomía Costarricense 39(2): 33-45. ISSN:0377-9424 / 2015 
resultados eran de esperarse, debido a que estos grupos raciales pertenecen a 2 subespecies diferentes (Bos taurus y Bos indicus). En este sentido es importante recalcar que, a pesar de que el número de marcadores es reducido, sí fue posible establecer una discriminación clara a nivel de las frecuencias alélicas entre las subpoblaciones de distintas especies y aún entre razas puras.

Los valores más bajos de distancias genéticas encontrados en este estudio son inferiores al 0,12 reportado por Brasil et ál. (2013) entre las razas Brahman vs. Nellore; así como el 0,10 reportado por Egito et ál. (2007) entre las razas Nellore vs. Gyr. Los valores más altos de distancias genéticas encontrados en este estudio son similares a los reportados por Brasil et ál. (2013) entre las razas Nellore vs. Holstein $(0,44)$; Nellore vs. Jersey $(0,48)$; Brahman vs. Holstein $(0,48)$; Gyr vs. Holstein $(0,49)$; Gyr vs. Jersey $(0,50)$ y Brahman vs. Jersey $(0,51)$, así como a los reportados por Egito et ál. (2007) entre las razas Holstein vs. Gyr $(0,34)$, Jersey vs. Gyr $(0,37)$, Nellore vs. Holstein $(0,38)$ o Nellore vs. Jersey $(0,38)$.

En el Cuadro 3 se presentan también estimados de diferenciación genética $R_{S T}$ (Slatkin $1995)$ entre todos los pares de razas con su significancia estadística. Para la mayoría de las subpoblaciones raciales, la diferenciación genética fue altamente significativa $(\mathrm{p}<0,001)$. En general se observó una buena correlación entre los valores de diferenciación genética $\left(R_{S T}\right)$ por pares de razas y las distancias genéticas de Nei.

Como era de esperarse, se observó una mayor diferenciación entre pares de diferente subespecie (Bos taurus vs. Bos indicus), mientras que se observó la menor diferenciación para grupos raciales más afines en origen. La menor diferenciación genética $\left(R_{S T}=0\right)$ se presentó entre las subpoblaciones Bos taurus $\times$ Bos indicus vs. Criollas para leche; Bos taurus $\times$ Bos indicus vs. Cruces para doble propósito; Criollas para doble propósito vs. Cruces para doble propósito; Pardo Suizo vs. Simmental. Además la diferenciación genética en estas subpoblaciones raciales no fue significativa $(p>0,05)$.
Cabe mencionar que los valores de $R_{S T}$ entre las subpoblaciones Bos taurus $\times$ Bos indicus vs. Criollas para leche; Pardo Suizo vs. Simmental fueron negativos $(-0,005$ y $-0,003$ respectivamente) y por tanto el programa los convierte a cero. Los valores negativos pueden ocurrir cuando la variación dentro de poblaciones es mayor que la variación entre poblaciones, que puede ser el caso en estas subpoblaciones ya que están conformadas por animales muy heterogéneos.

Por otra parte las subpoblaciones Bos indicus vs. Jersey $(0,17)$; Bos indicus vs. Holstein $\times$ Jersey $(0,18)$; Bos indicus vs. Holstein $(0,19)$ y Bos indicus vs. Guernsey $(0,20)$ presentaron la mayor diferenciación genética, al ser además, altamente significativa $(\mathrm{p}<0,001)$. Esto sugiere que estos grupos raciales pueden ser claramente discriminados mediante los 18 marcadores utilizados en el presente estudio.

A partir de las distancias estándar de Nei (1972), representadas en el Cuadro 3 se construyó un dendrograma con el algoritmo de Neighbor-Joining (Saitou y Nei 1987) (Figura 1a). Se observa la formación de 2 grupos principales, con las razas cebuinas (abajo) y las taurinas (arriba). También se observa cierta separación de las razas Simmental y Pardo Suizo con respecto a las demás razas taurinas. Los valores de confianza para las separaciones nodales, obtenidos por remuestreo, fueron muy variables entre un mínimo de 41 y un máximo de 100. Estos valores tienden a ser más altos para los nodos que separan razas puras, posiblemente porque su estructura genética está más claramente diferenciada.

Todos los grupos raciales que son genéticamente afines se encuentran en los mismos grupos, con la excepción del grupo racial Criollas para leche. Sin embargo, es posible que este grupo racial tenga una importante influencia de grupos raciales Bos indicus. En este sentido es importante recalcar, que el número de microsatélites empleados sí permitió establecer una discriminación clara a nivel de las frecuencias alélicas entre las razas puras. 
En la Figura 1b) se muestra un dendrograma para el cual se utilizó el método de agrupamiento de Ward (Ward 1963) sobre una matriz de distancia basada en el índice de similitud de Dice (Dice 1945). El análisis de conglomerados permitió clasificar básicamente 3 grupos de razas de acuerdo con la subespecie bovina y al sistema de producción a la que pertenecen. Un grupo lo conforman 5 subpoblaciones raciales, con mayor influencia Bos taurus y tradicionalmente lecheras de origen Europeo (Jersey, Holstein $\times$ Jersey, Holstein, Guernsey y Cruces para leche) localizados en la parte superior del dendrograma. Por otro lado, un grupo adicional lo conforman
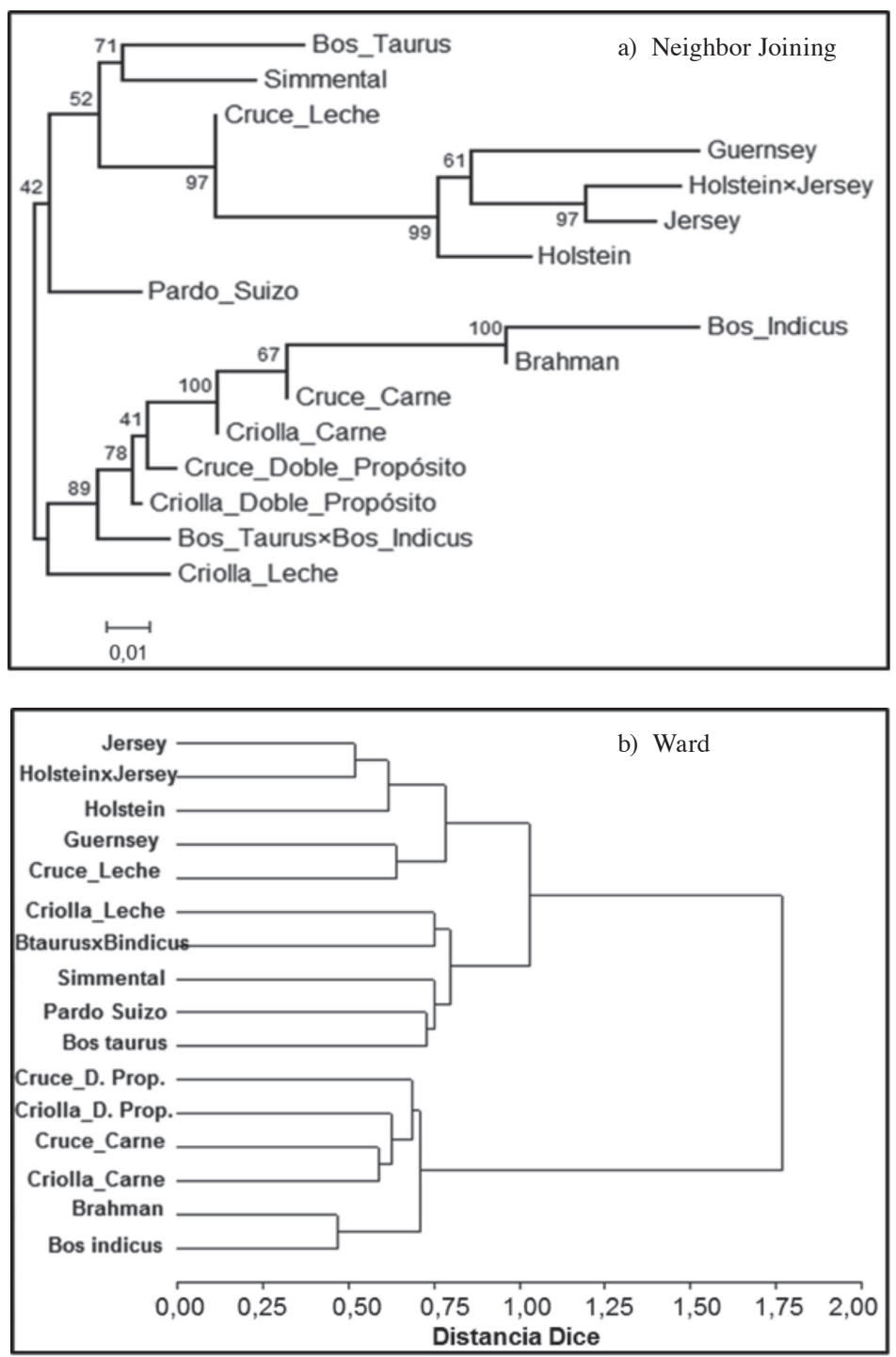

Fig. 1. Relaciones genéticas entre 16 subpoblaciones raciales bovinas de Costa Rica obtenidas por los algoritmos a) Neighbor Joining (Saitou y Nei 1987) (valores en bifurcaciones corresponden a niveles de confianza basados en 1000 iteraciones de remuestreo), y b) Ward (Ward 1963). 
5 subpoblaciones raciales con influencia $B o s$ taurus (Criollas para leche, Bos taurus $\times$ Bos indicus, Simmental, Pardo Suizo y Bos taurus) localizadas en una posición intermedia en el dendrograma. En este grupo, principalmente las razas Simmental, Pardo Suizo y Bos taurus han evolucionado como razas Doble Propósito de alta producción, por tanto forman una agrupación adicional.

Finalmente, un grupo lo conforman principalmente 6 subpoblaciones raciales con mayor influencia Bos indicus (Cruces para doble propósito, Criollas para doble propósito, Cruces para carne, Criollas para carne, Brahman y Bos indicus) localizados abajo del dendrograma. Es importante destacar que este subgrupo ha evolucionado históricamente como razas orientadas a la producción de carne, además de ser genéticamente más parecidos por ser originarias de la India.

Estas 3 grandes agrupaciones de razas representan a las subpoblaciones bovinas como las más diferentes, ya que se unen a la mayor distancia genética, debido a sus orígenes geográficos distintos y al sistema de producción.

Los dendrogramas también ratificaron que las denominaciones Criollo y Cruce dentro de un mismo sistema de producción, corresponden en realidad a animales con características genéticas muy similares. Las diferencias genéticas de estos mismos grupos entre los diferentes sistemas de producción (ej. Criollo Carne vs. Criollo Doble Propósito vs. Criollo Leche) está en función principalmente de las proporciones relativas de razas Bos indicus vs. Bos taurus presentes en el cruce.

\section{LITERATURA CITADA}

ACOSTA A.C., UFFO O., SANZ A., RONDA R., OSTA R., RODELLAR C., MARTIN I., ZARAGOZA P. 2012. Genetic diversity and differentiation of five Cuban cattle breeds using 30 microsatellite loci. Journal. Anim. Breed. Genet. 130(1):79-86.

APPLIED BIOSYSTEMS. 2002. ABI PRISM ${ }^{\circledR}$ GeneMapper ${ }^{\mathrm{TM}}$ Software Versión 3.0 User's Manual. 342 p. Consultado 24 oct. 2013. Disponible en http://www3.appliedbiosystems.com/cms/groups/ mcb_support/documents/generaldocuments/ cms_041304.pdf

BALZARINI M., DI RIENZO J. 2004. Info-Gen: Software para análisis estadístico genómico. Facultad de Ciencia Agropecuarias. Universidad Nacional de Córdoba. Argentina. Versión 2013. Disponible en http://www.info-gen.com.ar/

BRASIL B.S., COELHO E.G., DRUMMOND M.G., OLIVEIRA D.A. 2013. Genetic diversity and differentiation of exotic and American commercial cattle breeds raised in Brazil. Genetics and Molecular Research 12(4):5516-5526.

CAÑON J., ALEXANDRINO P., BESSA I., CARLEOS C., CARRETERO Y., DUNNER S., FERRAN N., GARCIA D., JORDANA J., LALOE D., PEREIRA A., SANCHEZ A., MOAZAMI K. 2001. Genetic diversity measures of local European beef cattle breeds for conservation purposes. Genet. Sel. Evol. 33:311-332.

CORDERO J.M., ROJAS G., VARGAS B., LEÓN B. 2013. Uso de marcadores moleculares para identificación y verificación de parentescos en ganado bovino. Informe Final. Proyecto FI-370-11 CONICIT. 16 p.

CORFOGA (CORPORACIÓN GANADERA, CR). 2000. Análisis de censo ganadero 2000. San José. Costa Rica. Consultado 30 jun. 2013. Disponible en http:// www.mag.go.cr/biblioteca_virtual_animal/censoganadero-2000.pdf

DICE L.R. 1945. Measures of the amount of ecologic association between species. Ecology 26(3):297302. Consultado 24 jun. 2013. Disponible en http:// biocomparison.ucoz.ru/_ld/0/86_dice_1945.pdf

EGITO A.A., PAIVA S.R., ALBUQUERQUE M DO S.M., MARIANTE A.S., ALMEIDA L.D., CASTRO S.R., GRATTAPAGLIA D. 2007. Microsatellite based genetic diversity and relationships among ten Creole and commercial cattle breeds raised in Brazil. BMC Genetics, pp. 1-14. Consultado 30 ene. 2014. Disponible en http://www.biomedcentral.com/ content/pdf/1471-2156-8-83.pdf

ESTOUP A., JARNE P., CORNUET J.M. 2002. Homoplasy and mutation model at microsatellite loci and their consequences for population genetics analysis. Mol Ecol. 11:1591-1604.

EXCOFFIER L., SMOUSE P., QUATTRO J. 1992. Analysis of molecular variance for metric distances among DNA haplotypes: application to human mitochondrial DNA restriction data. Genetics 131:479-491.

FAO (ORGANIZACIÓN DE LAS NACIONES UNIDAS PARA LA AGRICULTURA Y LA ALIMENTACIÓN, IT). 2011. Commission on genetic resources for food and agriculture. Draft guidelines on molecular genetic characterization of animal genetic resources. $13^{\text {th }}$ Regular Session. Rome, 2011. 70 p. 
FAO (ORGANIZACIÓN DE LAS NACIONES UNIDAS PARA LA AGRICULTURA Y LA ALIMENTACIÓN, IT). 2012. Commission on genetic resources for food and agriculture. Report of a consultation on the definition of breed categories. $7^{\text {th }}$ Regular Session. Rome, 2012. 7 p.

FELSENSTEIN J. 1985. Confidence limits on phylogenies: An approach using the bootstrap. Evolution 39:783791.

GINJA C., DA GAMA L.T., T PENEDO M.C. 2009. Analysis of STR Markers Reveals High Genetic Structure in Portuguese Native Cattle. Journal of Heredity 1-10.

HIDALGO C., MONGE C., CRUZ A., MOLINA J., CAMACHO J., VARGAS G., BARRIENTOS O. 2004. Informe preliminar de país sobre la situación nacional de los recursos zoogenéticos. Subcomisión nacional encargada de la elaboración del informe país sobre la situación de los recursos genéticos pecuarios de Costa Rica. Ministerio de Agricultura. San José, Costa Rica. p 43.

JORDANA J., ALEXANDRINO P., BEJA A., BESSA I., CAÑON J., CARRETERO Y., DUNNER S., LALOE D., MOAZAMI K., SANCHEZ A., FERRAND N. 2003. Genetic structure of eighteen local south European beef cattle breeds by comparative F-statistics analysis. J Anim Breed Genet. 120:73-87.

LIRÓN J.P., PERAL P., GIOVAMBATTISTA G. 2006. Genetic characterization of Argentine and Bolivian Creole cattle breeds assessed through microsatellites. Journal of Heredity 97(4):331-339.

NEI M. 1972. Genetic distances between populations. The American Naturalist 106:283-292. Consultado 22 set. 2013. Disponible en http://www.jstor.org/ stable/2459777

NEI M. 1977. F-statistics and analysis of gene diversity in subdivided populations. Ann. of Hum Genet. 41:225233.

PEAKALL R., SMOUSE P.E. 2006. GenAlEx 6: genetic analysis in Excel. Population genetic software for teaching and research. Mol. Ecol. Notes 6:288-295.

PEAKALL R., SMOUSE P.E. 2012. GenAlEx 6.5: genetic analysis in Excel. Population genetic software for teaching and research - an update. Bioinformatics 28:2537-2539. Consultado 24 jun. 2013. Disponible en http://bioinformatics.oxfordjournals.org/ content $/ 28 / 19 / 2537$

QUIRÓS E. 2006. Historia de la Ganadería Bovina en Costa Rica. CORFOGA. Consultado 30 jun. 2013. Disponible en http://www.corfoga.org/images/
public/documentos/pdf/Historia_Ganaderia_ bovina.pdf

SAITOU N., NEI M. 1987. The Neighbor-joining Method: A New Method for Reconstructing Phylogenetic Trees. Molecular Biology and Evolution. 4:406-425. Consultado 18 set. 2013. Disponible en http://homes. di.unimi.it/ re/Corsi/CB11mat/SaitNeiNJ.pdf

SIREA (SISTEMA INTEGRADO DE REGISTRO DE ESTABLECIMIENTOS AGROPECUARIOS, CR). 2013. SENASA (Servicio Nacional de Salud Animal, CR). Consultado 22 jun. 2013. Disponible en http://registrosenasa2.addax.cc/index. php? module $=$ Pagesetter $\&$ tid $=4$

SLATKIN M.A. 1995. Measure of population subdivision based on microsatellite allele frequencies. Genetics 139:457-462.

SUN W., CHEN H., LEI C., LEI X., ZHANG Y. 2008. Genetic variation in eight Chinese cattle breeds based on the analysis of microsatellite markers. Genetic Sel Evol. 40:681-692.

TAKEZAKI N., NEI M., TAMURA K. 2010. POPTREE2: Software for constructing population trees from allele frequency data and computing other population statistics with Windows interface. Mol Biol Evol. 27:747-752.

VILABOA J., QUIRÓS O., DÍAZ P., WINGCHING R., BROWER N., ZETINA P. 2012. Los sistemas ganaderos con Criollo Lechero Tropical (Reyna) en Costa Rica. Agronomía Mesoamericana 23(1):167-178

WARD J.H. 1963. Hierarchical Grouping to Optimize an Objective Function. Journal of the American Statistical Association 58:236-244.

WEIR B.S., COCKERHAM C.C. 1984. Estimating F-statistics for the analysis of population structure. Evolution 38(6):358-1370. Consultado 20 oct. 2013. Disponible en http://thirteen-01.stat.iastate.edu/ wiki/files?filename $=$ Weir1984.pdf

WRIGHT S. 1950. The genetical structure of populations. Annals of Human Genetics. Annals of Human Genetics 323-354. Consultado 20 oct. 2013. Disponible en http://onlinelibrary.wiley.com/ doi/10.1111/j.1469-1809.1949.tb02451.x/pdf

WRIGHT S. 1965. Interpretation of population structure by F-statistics with special regard to system of mating. Evolution 19:395-420. Consultado 20 oct. 2013. Disponible en http://www.jstor.org/ stable $/ 2406450$ ?seq $=2$

Todos los derechos reservados. Universidad de Costa Rica. Este artículo se encuentra licenciado con Creative Common Reconocimiento-NoComercial-SinObraDerivada 3.0 Costa Rica. Para mayor información escribir a rac.cia@ucr.ac.cr 
